\title{
Medicina del mejoramiento, ¿reto a los fines de la Medicina?
}

\section{Medicine of improvement, challenge to the purposes of Medicine?}

\author{
María de la Luz Casas Martínez*
}

https://doi.org/10.36105/mye.2020v31n3.03

\section{Resumen}

\begin{abstract}
La medicina del mejoramiento reta los fines tradicionales del acto médico basado en la terapéutica, y se acerca al pensamiento transhumanista. Actualmente se practican, con aval científico, diversas modalidades de medicina del mejoramiento, pero deben ser reflexionados sus extremos en sujetos sanos, a través del enhancement, a fin de no perder el sentido propio de la medicina y el cuidado de la beneficencia del paciente.
\end{abstract}

Palabras clave: medicina del mejoramiento, enhancement, transhumanismo, fines de la medicina, acto médico, bioética.

\section{Introducción}

El biomejoramiento humano (enhancement) ha dejado de ser una cuestión de ciencia ficción para ser ya una realidad en la medicina.

* Investigadora. Universidad Panamericana México, Centro Interdisciplinario de Bioética. México. Correo electrónico: mcasas@up.edu.mx https://orcid.org/00000002-47 22-6125

Recepción: 20 de marzo de 2020. Aceptación: 15 de mayo de 2020. 
El ejercicio médico contemporáneo incluye varias prácticas, cuyo objetivo no es el curar enfermedades, sino la mejora del individuo. Algunas de estas prácticas, aunque no terapéuticas en sí mismas, han demostrado un impacto directo en la calidad de vida de los sujetos, como es el caso de la medicina preventiva, cuidados paliativos, obstetricia, medicina del deporte, cirugía plástica, contracepción, tratamiento de la fertilidad, procedimientos cosméticos, entre otros. Estas prácticas se consideran estándares en la medicina actual y actúan bajo un marco diferente al de la medicina curativa; no proponen cambios extremos en el organismo o la conducta humana, pero sí funciones optimizadas o antes no existentes, como en el caso de la contracepción y técnicas de reproducción asistida.

Actualmente, este acervo de prácticas se ha diversificado cada vez más hacia la práctica del perfeccionamiento o mejoramiento en personas sanas. Se trata del uso de fármacos, cirugía y biotecnología, en algunos casos con resultados extremos.

El siguiente artículo se realizó a través del análisis, reflexión y propuesta de la literatura científica y representativa sobre el tema de la medicina del mejoramiento humano.

\section{Medicina del mejoramiento}

Por medicina del mejoramiento aceptamos la definición de Nick Bostrom: El mejoramiento es típicamente opuesto a la terapia. En términos sencillos, la terapia tiene como objetivo arreglar algo que está mal, curando enfermedades especificas o heridas, mientras que la intervención de mejoramiento tiene como objetivo impulsar el estado de un organismo a ser mejor que el considerado como un normal estado de salud. ${ }^{1}$

Existe una gran cantidad de ejemplos cotidianos de la medicina del mejoramiento, pero un ejemplo claro de ello es el uso del Sildenafil (Viagra) en jóvenes sanos, para potenciar su desempeño sexual. Muchas personas, algunas jóvenes, desean tener experiencias sexuales al límite. Estas personas no se conforman con una 
función normal; desean potenciar sus funciones al máximo, sin importar posibles riesgos, siempre y cuando existan resultados rápidos y evidentes en sus funciones, en este caso, el sexual.

Otro ejemplo es el auge en el uso de potenciadores cognitivos farmacológicos (PCF), como los inhibidores de modafinilo, metilfenidato y acetilcolinesterasa. Este consumo no terapéutico ha ocurrido principalmente en contextos académicos y laborales, y el interés del público en general ha aumentado significativamente, como señalan varios autores. ${ }^{2}$

Uno de los puntos relevantes también lo encontramos en la prescripción de antidepresivos, como la fluoxetina (Prozac), también utilizado sin una razón terapéutica concreta, sino como un potenciador del ánimo en una amplia población aparentemente sana o con leves trastornos psicológicos que, más que requerir farmacoterapia, serían candidatos a psicoterapia. Gran cantidad de personas sin problemas psicológicos serios experimentan en su vida contrariedades que podrían ser resueltas a través de actitudes positivas y actividades liberadoras de endorfinas endógenas; en su lugar, toman antidepresivos y no realizan ningún esfuerzo de reeducación de las emociones. Señala Célis ${ }^{3}$ que solamente el mercado mexicano para estos tratamientos equivale a cerca de 180 millones de dólares al año.

El abuso de este farmaco es ya reconocido ${ }^{4}$ científicamente, así como la necesidad de que su utilización responda a motivos terapéuticos concretos.

La cirugía estética es otra aplicación relativamente novedosa en el campo de la salud y se ha constituido como un negocio global. Se estima que en Estados Unidos esta industria genera entre 15 y 20 mil millones de dólares al año, ${ }^{5}$ situándose cerca de la cosmetología, con 25 mil millones anuales, y de los más de 30 mil millones anuales de la industria de las dietas. Ya en 2017, por ejemplo, la Sociedad Americana de Cirujanos Plásticos ${ }^{6}$ anunció que en el último año se habían llevado a cabo más de quince millones de cirugías plásticas, y la cifra se incrementa anualmente en más del 10\%. 
Así, la Sociedad Internacional de Cirugía Estética y Plástica señaló que el aumento de senos es un procedimiento frecuente (13\%), pero muy por debajo de las abdominoplastias (19\%), de la reconstrucción vaginal $(30 \%)$ y del implante de pectorales $(50 \%)$. Estas cirugías están cada vez más encaminadas a propósitos subjetivos personales que se alejan, no solamente de las indicaciones terapéuticas, sino de las recomendaciones hacia la seguridad del paciente, sin dejar de considerar los lineamientos estéticos universales.

Un ejercicio racional y necesario de los diversos campos del mejoramiento humano, como la cirugía plástica, cumple con los principios de la ética profesional, pero el ejercicio dedicado solamente a realizar fantasías de los pacientes, o a cirugías extremas (como, por ejemplo, realizar intervenciones para parecerse a un personaje imaginario o de la pantalla cinematográfica...) excede los fines propios de la medicina.

Los problemas psiquiátricos asociados pocas veces se mencionan o son estudiados por los mismos cirujanos. ${ }^{7}$ Una serie de estudios han sugerido constantemente un mayor riesgo de suicidio entre las mujeres que se han sometido a una cirugía estética de aumento de senos. Rohrich ${ }^{8}$ señala que existe un riesgo ligeramente mayor de suicidio (un poco más del doble que el encontrado en la población general) entre las mujeres con implantes mamarios.

Según el Manual Diagnóstico y Estadístico de los Trastornos Mentales, el Trastorno Dismórfico Corporal (TDC) se designa como un trastorno del Eje I, que se caracteriza por la intensa preocupación de un individuo con un defecto imaginario en su apariencia. Al igual que con muchos otros trastornos del Eje I, el individuo debe experimentar angustia significativa $y / o$ deterioro funcional. El TDC ocurre hasta en el uno por ciento de la población general, pero aumenta notablemente en personas que acuden compulsivamente a la cirugía plástica. ${ }^{9}$

El caso es que el uso de medicamentos y cirugías en personas sanas, especialmente jóvenes, llama mucho la atención en el campo de la bioética, pues son médicos los que proporcionan las recetas y 
la tecnología para su administración, en muchas ocasiones fuera de la indicación terapéutica.

Éstos son solamente unos cuantos ejemplos de la medicina del perfeccionamiento o enhancement, actos realizados por médicos, que se relacionan más con la medicina del deseo que con las propias necesidades de salud. ${ }^{10}$

El hecho es que la biotecnología ha posibilitado un nuevo fin de la medicina, la de ejercer posibles mejoras, además de la tradición terapéutica.

Es evidente, por tanto, que este tipo de intervenciones se encuentran posicionadas tanto en el sector social como en el profesional, y constituyen una nueva forma de actuar dentro del campo de la medicina.

Esta búsqueda de cambios perfectivos, así como de la ampliación de funciones y creación de nuevas aptitudes, lleva a reconsiderar los fines de la medicina y a una nueva relación médico/ paciente, basada en la aceptación por los médicos de los deseos subjetivos de sus clientes.

Aunque éstos son solamente unos breves ejemplos, claramente se aprecia que el hacer médico se ha expandido y que ahora considera actos que hasta hace poco no estaban al alcance de su hacer. La tecnología ha ampliado el campo del acto médico, pero en forma más importante también ha variado, para muchos profesionales, el fin propio de la medicina, pasando del campo de la necesidad terapéutica a la medicina del deseo de las personas, que ya no son pacientes, pues se encuentran en estado de salud.

Para poder diferenciar un acto médico de aquel desiderativo, es necesario definir el campo del primero.

\section{Los fines actuales de la medicina}

Durante más de veinticinco siglos se había considerado que la medicina tenía como objetivo y fundamento la cura de las enfermeda- 
des, la restauración de la salud y, más recientemente, la prevención de enfermedades y la rehabilitación. Los avances científicos y biotecnológicos, así como la incidencia de factores sociales y jurídicos en el mundo de la salud, han llevado, especialmente a la medicina, a reconsiderar su epistemología y praxis, ya que las expectativas emergentes sobrepasan los objetivos clásicos de la atención médica. El modelo unilateral de decisión terapéutica ha sido forzado al cambio por la incidencia de la multidisciplinariedad en las áreas de salud. Biotecnología, economía, política, derecho, filosofía, ${ }^{11}$ entre muchos otros campos del conocimiento, complementan la comprensión de los problemas, pero también hacen que estas disciplinas, debido a su complejidad, dificulten la toma de decisiones.

El reto de reformular cuáles podrían ser los nuevos fines de la medicina en la sociedad actual fue asumido en 1993 por el Hastings Center en Nueva York, bajo la dirección de Daniel Callahan, en el proyecto internacional titulado The Goals of Medicine. ${ }^{12}$

En dicho proyecto participaron expertos de 14 países. El grupo de trabajo definió los siguientes cuatro fines:

a) La prevención de la enfermedad y la promoción de la salud. Podemos desglosar el sentido contenido en esta frase. Prevenir no solamente es menos costoso que curar, sino que conservar la salud es la meta principal del ejercicio de la medicina. Esta intención no excluye el hecho de que somos seres vulnerables e imperfectos $y$, por tanto, no se niega la enfermedad y la muerte, por lo que este objetivo no es el foco único de la medicina.

b) El alivio del dolor y del sufrimiento causado por las enfermedades. Tradicionalmente, luchar contra el dolor y el sufrimiento ha sido aliciente para el desarrollo de la medicina, así como la atención humanística y el contacto entre personas forman parte del arte propio del ejercicio de la medicina.

c) El cuidado y curación de quienes padecen enfermedad y el cuidado de los que no pueden ser curados. El fin tradicional de la medicina se ha ampliado ahora hacia las acciones paliativas, al en- 
focarse a este importante campo, el cual cumple tanto con la ciencia como con el sentido humano de la misma.

d) Prevenir la muerte prematura y posibilitar una muerte en paz. La razón y la realidad nos muestran la imposibilidad de evitar la muerte, pues es el destino de todo ser vivo. La meta no es ésa, sino ayudar a enfrentar esa realidad con ausencia de los síntomas catastróficos que impidan a la persona tener una muerte tranquila. Estas acciones no significan eutanasia, sino el control de los síntomas físicos y psicológicos que producen sufrimiento extremo a la persona. La medicina paliativa es actualmente una de las acciones médicas más valoradas en las ciencias de la salud.

Pero estas interesantes conclusiones, apenas emitidas en el año 2000 , en muy pocos años han sido cuestionadas por la medicina del mejoramiento.

Para la visión tradicional, efectivamente los fines de la medicina en el siglo XXI deben ser algo más que la curación de la enfermedad y el alargamiento de la vida, pero teniendo en mente que el poder de la medicina no es absoluto. Para ello hay que acotar la tendencia, evidente en las sociedades desarrolladas, a medicalizar todos los problemas humanos.

La medicina actual dedica muchos de sus esfuerzos a la prevención; reduce la probabilidad de enfermedad y muerte a través de la vacunación y la medicina preventiva; a través de procesos que disminuyen el envejecimiento o de intervenciones para disminuir el riesgo de enfermedades o discapacidad.

Todo esto sigue siendo terapéutico, pues acepta la condición humana de vulnerabilidad $\mathrm{y}$, sin perder de vista la realidad de la muerte y la enfermedad, trata de evitar el acortamiento natural de la vida o la aparición de enfermedades. Un ejemplo de este tipo de acciones podría ser el uso del láser para evitar la ceguera o los lentes intraoculares, así como diversas prótesis que tienen como finalidad la recuperación de funciones normales, pero que no pretenden llevar a esos pacientes a tener capacidades extraordinarias. 
A la luz de sus posibilidades y de los problemas contemporáneos, ¿cuáles habrían de ser entonces las prioridades futuras del ejercicio de la medicina? Puesto que la condición humana es inseparable de la enfermedad, del dolor, del sufrimiento y, finalmente, de la muerte, una práctica médica adecuada a dichas condiciónes ha de empezar por aceptar la finitud humana, y enseñar o ayudar a vivir en ella para el beneficio mismo del paciente, y no dejarse arrastrar por un desarrollo tecnológico imparable. ${ }^{13}$

Habiendo reflexionado sobre los fines actuales de la medicina, ¿qué podemos plantear en relación con la medicina del mejoramiento?

\section{3. ¿Medicina del mejoramiento?}

¿Qué es el mejoramiento humano? ¿Siempre más es mejor? La utilización de fármacos y biotecnologías potenciadoras de funciones, como se señaló al principio de este artículo, es una realidad actual en la medicina, pero existen extremos que nos acercan a dilemas que deben ser analizados por la bioética, como sucede en los siguientes casos:

Neil Harbisson, presentado en los eventos de Tecnología, Entretenimiento y Diseño (TED), ${ }^{14}$ es un joven inglés de 32 años que padece acromatopsia, enfermedad congénita que sólo le permite ver la escala de grises. Mediante biotecnología le fue implantado en su cráneo, en la región occipital, una antena conectada a un chip, que traduce el color en sonido. Con este hecho, médicamente asistido, Harbisson ha adquirido funciones no naturales en el ser humano. De hecho, se considera un ciborg. A la luz de los fines de la medicina moderna, ¿cómo podría calificarse este hecho? ¿Constituye un acto médico toda aplicación tecnológica en el ser humano?

En dicho caso se trató de suplir una función faltante en el paciente por otra diferente, pero no fue en forma alguna una acción restauradora, pues la función primaria nunca se instaló. Se trató, 
por tanto, de que la persona tuviera una cualidad no percibida en humanos (correlacionar ondas de color con sonidos diferentes). Desde la perspectiva bioética, la autonomía del paciente fue el punto clave de decisión para obtener una función transhumana para la que, hasta ahora, no se conoce una aplicación práctica.

Un caso más radical lo encontramos en las cirugías extremas de Kalaca Skull, ampliamente conocido en medios de comunicación masiva. Se trata de un hombre colombiano, Eric Yeiner Hincapié Ramírez, quien se sometió a varias cirugías en las que se mutiló la nariz y las orejas, se pigmentó de negro la esclerótica y se cortó la lengua en forma bífida, para parecer una calavera. ${ }^{15}$ Estas acciones las realizó un médico. ¿Es ético considerar prioritaria la autonomía de los pacientes, aunque deseen la mutilación? ¿Es uno de los fines de la medicina considerar toda fantasía de las personas como peticiones racionales?

Nuevamente, la autonomía y los deseos subjetivos de esta persona fueron el punto a considerar por los profesionales que realizaron estos actos de mutilación. Desde la perspectiva ética, dañar órganos o funciones sanas es opuesto a la beneficencia, pero en este caso los profesionales valoraron con mayor peso la autonomía del cliente.

Tomando en cuenta la definición de medicina del mejoramiento, podríamos considerar ético uno de los casos, el de Harbisson, pero no el de Eric Yeiner. Éste es muy difícil justificarlo desde la razón práctica y menos todavía desde la terapéutica. Pero el hecho es que estos casos existen y no son los únicos, por lo que es obligada una profunda reflexión de hacia dónde se dirige la medicina actual.

\section{Sobre las solicitudes de los pacientes}

Las consideraciones de todo mejoramiento hacia funciones no relacionadas con la salud parten de una visión basada en una utopía positivista, en la que todo lo que puede hacerse, debe hacerse. Esta 
forma de pensamiento fue precisamente uno de los argumentos reflexivos de la bioética desde sus inicios, postulando precisamente que la ciencia debería aplicarse con conciencia. ${ }^{16}$

Los médicos, al servicio no de pacientes, sino de clientes, aceptarán y apoyarán los cambios propuestos por ellos, aun los más alejados de los fines médicos, y así estos profesionales de la salud se convertirán, no en una autoridad de prestigio por sus asesorías basadas en la evidencia científica y en la ética, sino en empleados altamente calificados al servicio de quien compre sus servicios.

El hecho es que en el ser humano todo cambio de mejora biológica llega a ser un problema moral, especialmente si es extremo, como señala Edmund Pellegrino en su libro Biotechnology and the Human Good. ${ }^{17}$

\section{El papel de la autonomía en la relación sanitaria actual}

En la medicina moderna el respeto a la autonomía ha significado un logro histórico en el ejercicio de una medicina más participativa y justa, pero, llevada al extremo, ha contribuido a la aparición de un poderoso movimiento ${ }^{18}$ que considera como un derecho todo deseo, racional o irracional de los individuos.

Muchos médicos a favor de este movimiento extremo consideran a quienes acuden a ellos como solamente clientes, y éstos últimos ven a los médicos sólo como proveedores de servicios; para ellos se trata solamente de una actividad comercial más.

Las razones por las cuales algunos clientes solicitan servicios extremos tienen su origen en valoraciones subjetivas porque, frente a ellos mismos o la sociedad, consideran estar en condiciones de desventaja. Como señala acertadamente E. Pellegrino, estas personas no pueden considerarse pacientes porque no tienen ninguna carencia con respecto a la norma o alguna enfermedad o condición discapacitante y considerarlos asi, medicalizaría cada faceta de la existencia bumana normal, por lo que es preferible considerarlos consumidores, esto es clientes. ${ }^{19}$ 
El comercio es el valor principal de este tipo de relaciones y se afecta por la publicidad. Uno de los fines de la publicidad es la venta de productos, proponiendo a los consumidores la búsqueda de la felicidad y de sus deseos, incluso de los más subjetivos e irracionales. En la relación cliente/proveedor sucede así, pero la medicina no tiene esos fines.

Un fin de la medicina no es lograr la felicidad de las personas atendiendo a sus deseos, porque frecuentemente éstos exceden a las expectativas realistas y ninguna mejora será suficiente para contenerlos, y porque, para el logro del bien de las personas, especialmente de los pacientes, muchas acciones médicamente indicadas no serían deseadas por el paciente, pero se realizan porque son evidentemente beneficiosas desde la perspectiva de la realidad científica, aunque ellas ocasionen cierto dolor o sufrimiento calculado a través de una adecuada ponderación de la balanza riesgo/ beneficio.

Señala nuevamente Pellegrino que es un becho que la satisfacción de los deseos personales, la libertad de elección y la «calidad de vida» se han convertido, para muchos, en derechos en una sociedad democrática. ${ }^{20}$ Pero ello siempre debe tener como medida la realidad y no la fantasía.

Actualmente imperan las fuerzas del mercado y, junto a una autonomía extrema, la medicina del mejoramiento y el enhancement, tienen un campo extenso de desenvolvimiento. Es posible que muchos médicos lo acepten, algunos por autoconvencimiento, otros por conveniencia o por realizar acciones seguras en pacientes que de otra forma acudirían a la clandestinidad. Poco a poco, todo ello cambiará en gran parte los fines tradicionales de la medicina y, desde el puno de vista ético, muchos se encontrarán en un grave conflicto de intereses.

Como apunta L. Kass, los médicos tendrán el aliciente de tener una clientela solvente y satisfecha, pues se ha dado solución a un aparente problema subjetivo. Todo esto, aceptado por el gremio médico, haría que quienes piensen lo contrario se encontrarían violando un nuevo contrato social entre médico y 
paciente. A la luz de todo ello, no puede ser ignorada la posibilidad de un serio conflicto de intereses por parte del médico. ${ }^{21}$

Bajo esta nueva visión, el que el médico se niegue a ciertas intervenciones consideradas por él como fuera de la práctica médica, se interpretaría como falta a la obligación de respetar la autonomía de los pacientes.

A este respecto, también comenta Ottois: Uno de los problemas es que el mejoramiento englobado en la esfera médica debe presentarse bajo la apariencia de terapia, lo cual produce consecuencias lamentables. Debido a la medicalización de la vida y a la necesidad de proteger a los médicos de una posible mala práctica, deberán modificarse las indicaciones sobre las drogas o procedimientos hacia una medicina del bienestar en personas sanas. ¿Podrá, entonces, la medicina del mejoramiento ser considerada una nueva «especialidad médica»? 22

Por tanto, para justificar las intervenciones perfectivas, los estados no patológicos tenderían a ser considerados trastornos o causas de angustia, para poder ser medicalizados.

\section{6. ¿Medicina del mejoramiento, transhumanismo y posthumanismo?}

La medicina del mejoramiento se encuentra ligada a la idea de transhumanismo.

Éste es un movimiento cultural y una filosofía de vida que defiende la posibilidad, el derecho y/o el deber de continuar y acelerar la evolución de la vida humana, más allá de sus limitaciones, a través de la ciencia y la tecnología. ${ }^{23}$

La medicina del mejoramiento es una forma de enhancement. Sencillamente, como señala Bostrom, el enhancement es típicamente contrapuesto a la terapia. ${ }^{24}$

Para lograr su objetivo, el pensamiento transhumanista requiere legitimizarse, poseer un acervo moral que lo respalde y que justifique sus objetivos. 
El Manifiesto Transhumanista $\mathrm{H}+{ }^{25}$ no solamente señala una opción, sino una obligación: la postura centrada en el cambio humano a través de la biotecnología aplicada a todas las esferas de la vida, especialmente a las naturales. Su intención es que se asuma el efecto transformador de la sociedad desde la biotecnología aplicada al derecho de autodeterminación biológica, de la medicina del mejoramiento, del enhancement y de la aceleración de la evolución humana.

El pensamiento transhumanista lleva de la mano al posthumanismo. El ser humano irá siendo perfeccionado poco a poco más allá de lo considerado «normal» para, posteriormente, constituirse a través de intervenciones genéticas, implantes, chips y diversas intervenciones biomecánicas en un ser de otra especie, más perfecto que el de la humana, y así es definido por Bostrom, una nueva especie, la posthumana. ${ }^{26}$

La estrategia del transhumanismo se apoya en el imperativo tecnológico. ${ }^{27}$ Se trata de una obligación moral, en la que el único límite es lo físicamente imposible.

Un argumento del porqué no toda la sociedad está a favor del enhancement en los seres humanos es que podría alterar nuestro entendimiento de la excelencia humana o nuestras prácticas sociales, señala Austriaco. ${ }^{28}$

Otro argumento que limita el enhancement es que la naturaleza humana es una realidad compleja, y no se puede determinar la excelencia del todo modificando las partes. Esto lo podemos constatar en la manipulación genética, en donde la variante alélica, beneficiosa en un ambiente, podría no serlo en otro, aumentándose o disminuyéndose en cada caso la proporción de la variante en el transcurso de generaciones, como ya se ha comprobado en investigaciones actuales. ${ }^{29}$

Para la visión transhumanista, la naturaleza humana se reduce a cualidades biofísicas manipulables, reduciendo al ser humano a calidad biológica, de la que esperan se desarrollen cualidades intelectuales y espirituales, y así lo señala Bostrom: ${ }^{30}$ la tecnología mejorará 
nuestra capacidad de apreciar buena literatura, de poder entender mejor al otro, de ser creativo...

\section{7. ¿Nuevos fines de la medicina?}

Mejorar es también un fin de la medicina, y el progreso de esta ciencia es también deseable y ético, pero habrá que reflexionar seriamente en las limitaciones del uso de los adelantos biotecnológicos, especialmente en personas sanas. Efectivamente, como señala Bostrom, ${ }^{31}$ la línea entre mejoramiento y terapia cada vez es más estrecha, pero la medicina se aboca precisamente a prevenir la enfermedad, conservar la salud o rehabilitarla, sin sobrepasar los límites de la normalidad. En algunos casos, como en el de la cirugía estética, las vacunas, reproducción asistida, medicina del deporte o los fármacos moduladores de la conducta, nos encontramos ante una medicina del mejoramiento aceptada social y médicamente, pero no se pretende con ello cambiar radicalmente al ser humano ni a las generaciones futuras.

Por medicina del mejoramiento se hace referencia, entonces, a acciones médicas, avaladas por la comunidad científica, como seguras e indicadas en este momento histórico. El enhancement es, entonces, la búsqueda de funciones inexistentes en los humanos, o en tal grado que exista una diferencia muy importante en la cantidad o calidad de las mismas respecto de las funciones normales.

La medicina del mejoramiento ha sido aceptada por gran parte de la profesión médica, y se ejerce sin límites, sin considerar el adecuado riesgo/beneficio ni responder a verdaderos problemas de salud de los pacientes. Esta filosofía se liga en forma importante a la aceptación del pensamiento transhumanista y posthumanista.

Es imperativo reflexionar sobre los propios fines de la medicina y no desatender los abusos que el conocimiento médico pueda tener sobre la salud física y mental de los pacientes y los consumidores. La medicina ejercida sin timón puede perder su individualidad, 
y convertirse solamente en una herramienta más de la subjetividad de los clientes y de las fuerzas del mercado, perdiendo su vocación universal al servicio del cuidado de la salud.

El médico debe abstenerse de participar en actos que solamente acaten las preferencias subjetivas de sus pacientes y no tengan una base realista de beneficencia. Ya el Hasting Center lo señaló: sería un error considerar la autonomía como un fin fundamental de la medicina por su excesivo individualismo. ${ }^{32}$

Existen valores morales no instrumentales que hay que conservar, porque representan la esencia propia del hombre, como es precisamente su actitud altruista y auto-formadora ante la necesidad, el dolor, el sufrimiento y la muerte. La vida perfecta no deja de ser una utopía, que aleja al ser humano del ejercicio de valores fundamentales.

Bajo las premisas del ejercicio de la medicina del mejoramiento y del enhancement ligado al pensamiento transhumanista, la relación médico/paciente queda restringida a un contrato comercial entre clientes y proveedores de servicios, frecuentemente desprovista de la calidad tradicional de la relación médico/paciente. Existe, por tanto, un gran riesgo en mercantilizar los actos médicos, debido principalmente a que ellos se llevan a cabo en los servicios privados de salud.

Erik Malmqvist señala acertadamente que las enfermedades incapacitan a las personas, no les permiten ser felices y reducen su autonomia, por lo que resulta una meta prioritaria su atención en el sentido de la justicia distributiva, y su primer objetivo sería, por tanto, el curar y prevenir enfermedades más que expandir capacidades a través del enhancement. ${ }^{33}$

\section{Conclusión}

La medicina del mejoramiento y el enbancement son prácticas cada vez más frecuentes en la comunidad médica. Potenciadores cogni- 
tivos, sexuales, cirugía estética extrema y otras intervenciones en sujetos sanos llevan al ejercicio de la llamada medicina del deseo.

Esta búsqueda de cambios perfectivos a través del ejercicio de la medicina, así como de la ampliación de funciones y de la creación de nuevas aptitudes humanas nos conducen a reconsiderar los fines de la medicina y a una nueva relación médico/paciente.

Hemos comentado el uso indebido de una gran cantidad de medicamentos diseñados terapéuticamente y utilizados por poblaciones no terapéuticas para lograr mejoras por razones frecuentemente subjetivas y alejadas de la buena práctica médica.

La medicina del mejoramiento es, sin duda, una primera fase de la aceptación del transhumanismo, por lo que propongo que se tenga mayor cuidado para distinguir los argumentos de «pendiente resbaladiza» que se utilizan en las exhortaciones emocionales, para llegar a una perspectiva más juiciosa sobre la agenda tecnológicamente impulsada para la mejora biomédica. Una forma de «pendiente resbaladiza» es aquella en la que un acto particular con carga negativa moral o médicamente puede parecer inocuo a la hora de su aplicación individual pero, al dar luego origen a una serie de esos actos, constituirían un daño moral o científico evidente. La despenalización del aborto y la eutanasia son casos clásicos para ilustrar los argumentos de «pendiente resbaladiza» en la discusión pública y en la formulación de políticas públicas. La utilización de medicamentos para aumentar funciones cuantitativas y cualitativas en sujetos sanos es, sin duda, un paso hacia la promoción de cambios cada vez más drásticos e invasivos en los sujetos, llevándolos a la búsqueda de experiencias y funciones fuera de la naturaleza humana.

La medicina del mejoramiento, llevada al extremo, liga su pensamiento al transhumanismo y al post-posthumanismo, cuya propuesta es reconstruir en el futuro a la especie humana a través de intervenciones genéticas, sustancias químicas, implantes, chips y 
diversas intervenciones biomecánicas en un ser de otra especie, más perfecto que el humano, y así es definido por Bostrom: una nueva especie, la posthumana.

Como científicos y sujetos éticos, no estamos en contra del progreso biotecnológico, ni de la aplicación del mismo para lograr bienes racionales en la humanidad, pero las justificaciones subjetivas para cambios extremos, muchas veces con efectos adversos importantes, no son funciones propias de la medicina, la cual debe actuar en una base realista apegada a los fines propios de esta ciencia.

La medicina no tiene como objetivo «hacer felices a las personas», sino promover la salud.

Es necesaria la reflexión, a fin de que los médicos se nieguen a intervenciones basadas en la fantasía o puedan ir en contra de la buena práctica médica, para salvaguardar los verdaderos fines de esta profesión, cuya fidelidad a ellos es indispensable en el futuro de la humanidad.

\section{Notas bibliográficas}

${ }^{1}$ Bostrom, Nick, RoAche, Rebecca. Ethical Issues in Human Enhancement. En: New Waves in Applied Ethics. Eds. Jesper Ryberg, Thomas Petersen \& Clark WOLF (Pelgrave Macmillan): England; 2008; 120-152.

2 TeTER Smith, M. E. \& FARAH, M. J. Are prescription stimulants «smart pills»? The epidemiology and cognitive neuroscience of prescription stimulant use by normal healthy individuals. Psychol Bull; 2011; 137(5): 717-741. https://doi.org/10.1037/a 0023825

3 CÉLIS, D. Venta de antidepresivos en México supera los 180 mdd anuales en 2015. Excelsior. Consultado el 9 de marzo de 2019. Disponible en: https://www. excelsior.com.mx/opinion/2011/12/08/dario-celis/7927943

${ }^{4} \mathrm{KIRSCH}$, I., SAPIRSTEIN, G. Lisent Prozac, but listen to placebo: a metaanalysis of antidepressants drugs. En: I. KIRSCH (Ed.). How expectancies shape experience. 1999; 303-320. https://doi.org/10.1037/10332-012

${ }^{5}$ Elliot, Anthony. Anagramas. Medellín, Colombia. Volumen 9, № 18, pp. 145-164.

${ }^{6}$ American Society of Plastic Surgery. Noticias. Consultado 18 de marzo de 2020. Disponible en: https://www.plasticsurgery.org/

7 SANSONE, R. A., SANSONE, L. A. Aesthetic surgery and psychological problems. Psychiatry (Edgmont). Diciembre de 2007; 4(12): 65-8. PMID: 20436768; PMID: PMC2861519. 
${ }^{8}$ Rohrich, R. J., AdAms, W. P., POTTER, J. K. Una revisión de los resultados psicológicos y el suicidio en el aumento de senos estético. Plast. Reconstr. Surg. 2017; 119: 401-408.

${ }^{9}$ KREBS, GEORGINA ET ALT. Recent advances in understanding and managing body dysmorphic disorder. Evid Based Mental Health. August 2017; 20(371): 72. https:// doi.org/10.1136/eb-2017-102702

10 SCHLEIM, S., QUeDNOW, B. B. How realistic are the scientific assumptions of the debate on neurological improvement? Evaluation of hypotheses of neurostimulation prevalence and pharmacological optimism. Front. Pharmacol. 2018: 9(3) https://doi.org/10.3389 / fphar.2018.00003

${ }^{11}$ Nancarrow, S. A., Stand, A., Ariss, S., Smith, T., Enderby, P., Roots. A. Ten principles of good interdisciplinary team work. Hum Resour Health. 10 de mayo de 2013; 11: 19. https://doi.org/10.1186/1478-4491-11-19. PMID: 23663329; PMID: PMC3662612.

12 Hanson, M., CallahAN, D. Los objetivos de la Medicina. Los problemas olvidados en la reforma de la atención médica. Hastings Center Studies. En: Ethics Series. United States; 2000. https://doi.org/10.4067/s0034-98872003000900016

${ }^{13}$ CASAS, MARÍA DE LA LUZ. Bases Bioéticas para la toma de decisiones. Ed. Trillas: México; 2010, p. 32.

${ }^{14}$ TED. El renacimiento de nuestra especie. Consultado 28 de marzo de 2018. Disponible en: https://www.youtube.com/results?search_query=ted+talks+neil+harbisson

15 GutiÉRREZ, L. Entrevista Kalaca Scull, 2018. Consultado 25 de marzo de 2019. Disponible en: https://www.youtube.com/watch?v=gUmBxFGhUfk\&t=5s

${ }^{16}$ Polaino-Lorente, Aquilino. La dignidad de la persona. En Polaino-LORENTE, AQUILINO. Algunas cuestiones en torno a la fundamentación de la Bioética. 1997, $3^{\text {a }}$ ed., p. 27. https://doi.org/10.5585/prismaj.v9i2.2398

${ }^{17}$ Pellegrino, Edmund. Biotechnology and the Human Good. Georgetown University Press: United States; 2007

18 ThOMAS ANDREOLI, E. The undermining of academic medicine. ProQuest: Washington; tomo 85, No. 6, Nov/Dec 1999; pp. 32-37.

19 Pellegrino, E. Biotechnology, enhancement and goals of Medicine. Dignity. 2004, Vol 10, No. 4. Consultado 2 de marzo de 2019. Disponible en: https://cbhd. org/content/biotechnology-human-enhancement-and-ends-medicine

${ }^{20}$ Op. cit.

${ }^{21}$ KASS LEON, R. Regarding the End of Medicine and the Pursuit of Health. Public Interest: USA; 1995, 40: II. PMID:11662217

22 OTtoIs, GILBERT. Humanismo, Transhumanismo, Posthumanismo. Rev. Col. Bioet. 2013; 8(2): 7. https://doi.org/10.18270/rcb.v8i2.797

${ }^{23}$ Bostrom, Nick. A history of transhumanist thought. In Academic Writing Across the Disciplines. Eds. Michael Rectenwald \& LiSA CARL. New York: Pearson Longman; 2011, p. 15. 
Medicina del mejoramiento, ¿reto a los fines de la Medicina?

${ }^{24}$ Bostrom, Nick. Why I want to be a Posthuman When I Grow Up. Medical Enhancement and Posthumanity. Eds. BERT GoRDIJN AND RUTH CHADWICK: USA, Springer; 2008; p. 107. https://doi.org/10.1007/978-1-4020-8852-0_8

${ }^{25}$ HUMANITY + BOARD. Transhumanist Declaration. Consultado 18 de marzo de 2020. Disponible en: https://humanityplus.org/philosophy/transhumanist-declaration/

${ }^{26}$ Bostrom, N. In defense of posthuman dignity. Bioethics. 2005; 19(3): 202-214. https://doi.org/10.1111/j.1467-8519.2005.00437.x

27 JONAS, H. El principio de responsabilidad: ensayo de una ética para la civilización tecnológica. Barcelona: Herder; 1995. https://doi.org/10.2307/j.ctvt9k2sz

${ }^{28}$ AUSTRIACO, N. Human nature as normative concept: relevance for health care. En: Handbook of the philosophy of medicine. Dordrecht, Springer Science+ Business Media. 2015, pp. 1-10.

${ }^{29}$ HeRRERA-PAZ, EdWIN Francisco. La genética de poblaciones y el origen de la diversidad humana. 2013; vol. 81(1).

30 Bostrom, Nick, RebecCA, RoACHE. Ethical Issues in Human Enhancement. New Waves in Applied Ethics. Eds. Jesper Ryberg, ThOmas Petersen \&. Clark WOLF. 2008; p.137.

${ }^{31}$ Bostrom, Nick, RoAche, RebeccA. Ethical issues in human enhancement. En: J. Ryberg, T. Petersen y C. Wolf (eds.). New Waves in Applied Ethics. Palgrave-Macmillan; 2008, pp. 120-152

32 HASting CEnTER. Los fines de la Medicina. Ed. Cuadernos de la Fundación Víctor Grífols i Lucas. Barcelona; 2005.

${ }^{33}$ MALMQVIST, E. Reproductive choice, enhancement, and the moral continuum argument. J. Med. Philos. 2014; 39(1): 41-54. https://doi.org/10.1093/jmp/ jht058 
